\title{
Ertugliflozin in Patients with Stage 3 Chronic Kidney Disease and Type 2 Diabetes Mellitus: The VERTIS RENAL Randomized Study
}

\author{
George Grunberger · Sarah Camp · Jeremy Johnson · Susan Huyck • \\ Steven G. Terra · James P. Mancuso · Zhi Wei Jiang • Gregory Golm • \\ Samuel S. Engel · Brett Lauring (D)
}

Received: October 6, 2017 / Published online: November 20, 2017

(C) The Author(s) 2017. This article is an open access publication

\begin{abstract}
Introduction: Ertugliflozin is a sodium-glucose cotransporter 2 inhibitor in development for type 2 diabetes mellitus (T2DM). The safety and efficacy of ertugliflozin were evaluated over 52 weeks in patients with chronic kidney disease (CKD).
\end{abstract}

Methods: In this double-blind randomized study (NCT01986855), patients with glycated hemoglobin (A1C) $7.0-10.5 \%$ and stage 3 CKD

Enhanced content To view enhanced content for this article go to http://www.medengine.com/Redeem/ E2DCF060423E35E0.

Electronic supplementary material The online version of this article (https://doi.org/10.1007/s13300017-0337-5) contains supplementary material, which is available to authorized users.

\section{G. Grunberger}

Grunberger Diabetes Institute, Bloomfield Hills, MI, USA

S. Camp · J. Johnson · S. Huyck · G. Golm •

S. S. Engel · B. Lauring $(\bowtie)$

Merck \& Co., Inc., Kenilworth, NJ, USA

e-mail: brett_lauring@merck.com

S. G. Terra

Pfizer Inc., Andover, MA, USA

J. P. Mancuso

Pfizer Inc., Groton, CT, USA

Z. W. Jiang

MSD China, Beijing, China [estimated glomerular filtration rate $\left(\right.$ eGFR) $\geq 30$ to $<60 \mathrm{~mL} / \mathrm{min} / 1.73 \mathrm{~m}^{2}$ ] who were undergoing treatment with standard diabetes therapy (or therapies) including insulin and/or sulfonylureas were randomized to once-daily ertugliflozin $5 \mathrm{mg}, 15 \mathrm{mg}$, or placebo. Patients on metformin underwent a pre-randomization $\geq 10$-week wash-off period. The primary endpoint was change from baseline in A1C at week 26 in the overall cohort. Secondary efficacy endpoints were assessed in the stage $3 \mathrm{~A}$ CKD cohort (eGFR $\geq 45$ to $<60 \mathrm{~mL} / \mathrm{min}$ / $1.73 \mathrm{~m}^{2}$ ) at weeks 26 and 52 . Safety was assessed in the overall cohort.

Results: 468 patients were randomized (baseline mean A1C 8.2\%). At week 26, reductions from baseline in A1C were observed across groups in the overall cohort [least squares mean changes $(95 \%$ confidence interval) $-0.3 \%$ $(-0.4,-0.1),-0.3 \%(-0.4,-0.1)$, and $-0.4 \%$ $(-0.6,-0.3)$ for placebo and for ertugliflozin $5 \mathrm{mg}$ and $15 \mathrm{mg}$, respectively]. Prohibited use of metformin was identified in $\sim 17 \%$ of patients and impacted evaluation of the primary endpoint. Greater reductions from baseline in body weight, fasting plasma glucose, and systolic blood pressure were observed with ertugliflozin versus placebo at week 26 (stage 3A CKD cohort). The incidences of urinary tract infections, genital mycotic infections, and hypoglycemia adverse events were not meaningfully different between groups. The incidence of 
hypovolemia-related adverse events was higher with ertugliflozin relative to placebo.

Conclusion: Although surreptitious metformin use impacted the primary analysis, reductions in blood glucose and body weight were observed with ertugliflozin in patients with T2DM and stage 3 CKD; ertugliflozin had an acceptable safety profile.

Funding: Merck Sharp \& Dohme Corp. a subsidiary of Merck \& Co., Inc., Kenilworth, NJ, USA and Pfizer Inc.

Trial Registration: Clinicaltrials.gov identifier NCT01986855.

Keywords: Chronic kidney disease; Ertugliflozin; Glycemic control; SGLT2 inhibitor; Type 2 diabetes

\section{INTRODUCTION}

In patients with type 2 diabetes mellitus (T2DM) and hyperglycemia, the amount of glucose filtered by the kidney is increased [1]. While at least $90 \%$ of the filtered glucose is typically reabsorbed in patients with T2DM [2], sodium-glucose cotransporter 2 (SGLT2) inhibitors prevent reabsorption of filtered glucose, leading to urinary glucose excretion (UGE) and a consequent reduction in plasma glucose [3]. Due to their mechanism of action, the glycemic efficacy of SGLT2 inhibitors is dependent on renal function and the amount of filtered glucose [4]. As the glomerular filtration rate declines in patients with impaired renal function, the glycemic efficacy of SGLT2 inhibitors is attenuated, although beneficial effects on body weight and blood pressure (BP) have been observed [4-7]. Recent evidence suggests that SGLT2 inhibitors may have long-term beneficial effects on renal outcomes [8,9], which is likely due to several direct and indirect effects on the kidney through tubuloglomerular feedback and improvements in hyperglycemia, hypertension, obesity, and hyperuricemia [1, 10].

Ertugliflozin is a SGLT2 inhibitor that is highly selective for SGLT2 over other glucose transporters [11]. Ertugliflozin is being developed as an antihyperglycemic agent (AHA) for patients with T2DM in the VERTIS clinical program. The current study (VERTIS RENAL) was intended to evaluate the efficacy and safety of ertugliflozin in patients with T2DM and stage 3 chronic kidney disease [CKD; characterized by an estimated glomerular filtration rate (eGFR) $\geq 30$ to $<60 \mathrm{~mL} / \mathrm{min} / 1.73 \mathrm{~m}^{2}$ ]. Although there are differences in various regulatory jurisdictions around the world, current product labeling for approved SGLT2 inhibitors generally allows for the use of some doses in patients with eGFR values $\geq 45$ to $<60 \mathrm{~mL} / \mathrm{min} / 1.73 \mathrm{~m}^{2}$ [12-17]. A priori, it was expected that the efficacy of ertugliflozin would be greater in patients with stage $3 \mathrm{~A}$ CKD (eGFR $\geq 45$ to $<60 \mathrm{~mL} / \mathrm{min} / 1.73 \mathrm{~m}^{2}$ ) relative to patients with stage $3 \mathrm{~B}$ CKD (eGFR $\geq 30$ to $<45 \mathrm{~mL} / \mathrm{min} / 1.73 \mathrm{~m}^{2}$ ). Similar observations have been noted with other SGLT2 inhibitors. Therefore, the primary evaluation of efficacy in this study was conducted in the overall stage 3 CKD cohort, with additional secondary analyses conducted in the stage $3 \mathrm{~A}$ CKD cohort; the primary evaluation of safety was in the overall cohort.

\section{METHODS}

\section{Study Design}

The study (protocol MK-8835-001; VERTIS RENAL; ClinicalTrials.gov identifier: NCT01986855) was a randomized, double-blind, placebo-controlled, parallel-group phase 3 trial. The study was conducted at 121 centers across 13 countries (Argentina, Bulgaria, Colombia, Hungary, Israel, Mexico, Philippines, Poland, Romania, Russia, South Africa, United Kingdom, United States). All procedures followed were in accordance with the ethical standards of the responsible committee on human experimentation (institutional and national) and with the Helsinki Declaration of 1964, as revised in 2013. It was conducted in accordance with the principles of good clinical practice and approved by the appropriate institutional review boards and regulatory agencies. All participating patients provided written, informed consent. The protocol and statistical analysis plan were developed by the sponsors in consultation with an external scientific advisory committee. 
The duration of follow-up was 54 weeks and included a 26-week phase A, followed by a 26week phase $B$ extension treatment period, followed by a 2-week post-treatment assessment (Fig. S1 in the Electronic supplementary material, ESM). The trial started on 3 December 2013; the last patient completed phase A on 11 March 2016 and phase A + B on 28 September 2016. The time point for primary analysis was week 26 .

Randomization was stratified based on the visit 3 (week -2) eGFR measurement (stage 3A CKD stratum: eGFR $\geq 45$ to $<60 \mathrm{~mL} / \mathrm{min} /$ $1.73 \mathrm{~m}^{2}$; stage $3 \mathrm{~B}$ CKD stratum: eGFR $\geq 30$ to $<45 \mathrm{~mL} / \mathrm{min} / 1.73 \mathrm{~m}^{2}$ ), the presence or absence of a history of cardiovascular disease or heart failure, and the presence or absence of treatment with insulin at randomization. Randomization was implemented centrally using an interactive voice response system/integrated web response system. Eligible patients were randomized using a computer-generated schedule, with a block size of six. Ertugliflozin and placebo were packaged identically so that blinding was maintained. Patients, investigators, sponsor, and contract research organization personnel were blinded to group assignments during the 26week phase A part of the study. The sponsor was unblinded after the week 26 database lock to permit the writing of the phase A clinical study report. Personnel associated with the conduct of the study at the contract research organization, trial site personnel, and patients remained blinded until after the phase B portion of this study (26-week treatment and 14-day post-treatment follow-up) was completed.

\section{Patient Population}

The study enrolled patients $\geq 25$ years of age with T2DM according to American Diabetes Association guidelines [18], stage 3 CKD (eGFR $\geq 30$ and $<60 \mathrm{~mL} / \mathrm{min} / 1.73 \mathrm{~m}^{2}$ calculated using the MDRD equation) with stable renal function, and glycated hemoglobin (A1C) $7.0-10.5 \%$ on diet/exercise with or without AHA monotherapy or combination therapy using other AHAs including insulin and sulfonylureas. The only prohibited background AHAs were metformin, rosiglitazone, and other SGLT2 inhibitors. Patients on metformin at the screening visit were eligible to participate in the trial if their $\mathrm{A} 1 \mathrm{C}$ was $\geq 6.5 \%$ and $\leq 10.0 \%$; however, they were required to undergo $\mathrm{a} \geq 10$-week metformin wash-off, and they remained eligible if their $\mathrm{A} 1 \mathrm{C}$ was $\geq 7.0 \%$ and $\leq 10.5 \%$ at the end of this period. Metformin wash-off was required because at the time of trial initiation, metformin product labels generally did not recommend their usage in patients with moderate renal impairment. Stable renal function was defined as a change in eGFR $<10 \mathrm{~mL} / \mathrm{min} / 1.73 \mathrm{~m}^{2}$ between screening and visit 3 (week -2), with eGFR measurement $\geq 30$ to $<60 \mathrm{~mL} / \mathrm{min} / 1.73 \mathrm{~m}^{2}$ at both visits.

Key exclusion criteria included history of type 1 diabetes mellitus, history of ketoacidosis, renal-related medical history (including nephrotic range proteinuria (>3000 mg/day) with hypoalbuminemia and edema, rapidly progressive glomerulonephritis, lupus nephritis, renal or systemic vasculitis, renal artery stenosis with renovascular hypertension, or ischemic nephropathy, familial renal glucosuria, renal dialysis, renal transplant, or renal disease requiring treatment with immunosuppressive agents), active obstructive uropathy, or an indwelling urinary catheter.

Prior to randomization, eligible patients entered a 2-week single-blind placebo run-in period. Patients with adequate compliance ( $\geq 80 \%$ based on pill count) were randomized 1:1:1 to ertugliflozin $5 \mathrm{mg}$, ertugliflozin $15 \mathrm{mg}$, or placebo while continuing a diet/exercise regimen and background AHA therapy (if applicable); all blinded study treatments were taken once daily. Following completion of the initial 26-week treatment period, patients entered a 26-week placebo-controlled extension treatment period (phase $\mathrm{B}$, where they continued with their assigned randomized treatment from phase A); the aim of phase B was to gather additional data on the safety and longer-term efficacy of ertugliflozin. A post-treatment visit was scheduled 14 days after the last treatment dose to assess renal function and key safety parameters.

Patients who met progressively stricter protocol-defined glycemic rescue criteria [fasting plasma glucose (FPG) values consistently (repeat measurement performed within 3-7 days) $>$ $270 \mathrm{mg} / \mathrm{dL}$ after randomization through week 
$6 ;>240 \mathrm{mg} / \mathrm{dL}$ after week 6 through week $12 ;>200 \mathrm{mg} / \mathrm{dL}$ after week 12 through week $26 ;>200 \mathrm{mg} / \mathrm{dL}$ or $\mathrm{A} 1 \mathrm{C}>8.0 \%$ after week 26] were permitted to have an adjustment in the dose(s) of background AHA therapy or the addition of new AHA therapy (other than metformin or other prohibited AHAs). Rescued patients continued with the same dose and regimen of their study medication.

\section{Efficacy Assessments}

The primary time point for efficacy analysis was week 26 . The primary efficacy endpoint was the change from baseline in A1C at week 26 in the overall cohort. Key secondary efficacy endpoints, analyzed in the stage $3 \mathrm{~A}$ CKD cohort at week 26, were changes from baseline in $\mathrm{A} 1 \mathrm{C}$, body weight, systolic blood pressure (SBP), and FPG, as well as the proportion of patients with $\mathrm{A} 1 \mathrm{C}<7.0 \%$. Other efficacy endpoints are described in the ESM.

Efficacy assessments (A1C, FPG, body weight, $\mathrm{BP})$ were performed at weeks $0,6,12,18$, and 26 during phase $\mathrm{A}$, as well as at weeks 34,42 , and 52 during the phase $\mathrm{B}$ extension period. Body weight was measured with a standardized digital scale at study visits. Weight was measured in duplicate at the same time of day after voiding and while wearing only a gown (or light clothing) and underwear. Sitting BP was measured in triplicate at study visits using an automated oscillometric BP measuring device. Site personnel were instructed to use the same device for each patient throughout the study. Blood samples were collected for pharmacokinetic (PK) analysis of plasma concentrations of ertugliflozin at weeks 6, 12, and 18 .

Laboratory assessments were performed at a central laboratory where analysts were blinded to treatment assignment. Central laboratory tests were performed after an overnight fast of at least $8 \mathrm{~h}$ duration.

\section{Safety Assessments}

Safety endpoints included adverse events (AE), including pre-specified AEs and collections of AEs of special interest [symptomatic hypoglycemia and AEs associated with genital mycotic infection (GMI) (gender-specific), urinary tract infection, and hypovolemia]. In addition to symptomatic hypoglycemia, episodes of documented hypoglycemia, defined as episodes with a glucose level $\leq 70 \mathrm{mg} / \mathrm{dL}$ with or without symptoms, were also recorded. Pre-defined limits of change (PDLC; criteria based on normal ranges and abnormalities considered clinically meaningful) for pre-specified laboratory and electrocardiogram (ECG) parameters, as well as changes over time in laboratory parameters [including eGFR, low-density lipoprotein cholesterol (LDL-C) and high-density lipoprotein cholesterol (HDL-C)], ECG measurements, and vital signs were assessed.

Safety analyses were performed for the phase A (26-week treatment period) and phase A + B (52-week treatment period) periods. Analysis of the post-treatment eGFR change from baseline was performed in patients in the overall cohort who were on study medication at week 52 and had eGFR results at baseline, week 52 and week 54. Renal function was further evaluated through urinary albumin/creatinine ratio (UACR) at week 26. Normal albuminuria, microalbuminuria, and macroalbuminuria were defined as $\mathrm{UACR}<30, \geq 30$ and $\leq 300$, and $\mathrm{UACR}>300$, respectively.

\section{Statistical Analyses}

The planned sample size of 468 patients (156 per group) was estimated to provide $90 \%$ power to detect a true difference of $0.38 \%$ in the mean change from baseline in A1C between a given ertugliflozin dose and placebo, based on a two-sided test at a 5\% level of significance, assuming a dropout rate of approximately 13\%.

Efficacy analyses included all randomized, treated patients who had at least 1 measurement of the respective endpoint. Post-rescue efficacy data were treated as missing in efficacy analyses. A longitudinal data analysis (LDA) model [19] was used to evaluate continuous endpoints, with fixed effects for treatment, eGFR stratum (only for the primary hypothesis), baseline treatment with insulin stratum, time, 
and the interaction of time with treatment. Missing outcome data were handled implicitly by the model and not imputed. Logistic regression was used to evaluate the proportion of patients with $\mathrm{A} 1 \mathrm{C}<7.0 \%$, fitted with terms for treatment, baseline treatment with insulin stratum, and baseline A1C, with missing data imputed via multiple imputation based on the LDA model used for the primary analysis. The primary and key secondary efficacy endpoints were to be assessed using an ordered testing procedure (ESM). No hypothesis testing for efficacy endpoints was performed at week 52 .

Safety analyses included all randomized treated patients. Data following the initiation of glycemic rescue therapy were excluded for the analysis of hypoglycemia to avoid the confounding influence of the rescue therapy. All other safety analyses included data following the initiation of glycemic rescue therapy. $P$ values and 95\% confidence intervals (CI) for between-group differences in pre-specified endpoints were computed using the Miettinen and Nurminen method [20]. LDL-C and HDL-C were assessed by a LDA model similar to that used for the primary endpoint. Changes from baseline in eGFR were descriptively summarized.

\section{Post-Hoc Analyses}

Following the completion of phase A, investigation of retained plasma samples indicated that approximately $17 \%$ of the patients in each treatment arm used metformin-a prohibited medication-during the study and did not report the use to the investigators. This is in contrast to the protocol-specified use of hyperglycemic rescue medication (defined as the addition of a new AHA or the intensification of an existing AHA in those meeting the rescue criteria) which was reported to investigators. Metformin concentrations were assayed in retained PK or archived samples drawn at each post-baseline study visit (weeks 6, 12, 18, and 26; Table S1 in the ESM). Post-hoc analyses were performed to evaluate the treatment response in two subgroups: (1) patients who tested positive for metformin use at any time point and (2) patients who did not test positive for metformin use.

\section{RESULTS}

\section{Patient Disposition and Baseline Characteristics}

In total, 468 patients were randomized and 467 were treated (Fig. S2 in the ESM); 417 (89.1\%) completed phase A and 388 (82.9\%) phase B; discontinuations were balanced across treatment groups.

The baseline demographics of the treatment groups were similar (Table 1). The mean age was 67.3 years and the mean eGFR was $46.6 \mathrm{~mL} /$ $\mathrm{min} / 1.73 \mathrm{~m}^{2}$ in the overall cohort (stage $3 \mathrm{~A}$ CKD: $50.9 \mathrm{~mL} / \mathrm{min} / 1.73 \mathrm{~m}^{2}$; stage 3B CKD: $38.7 \mathrm{~mL} / \mathrm{min} / 1.73 \mathrm{~m}^{2}$ ). The mean duration of T2DM was 14.2 years and the mean $\mathrm{A} 1 \mathrm{C}$ was $8.2 \%$. More than $95 \%$ of the patients in each treatment group were on background AHA therapy at screening. Most patients (96\%) were receiving insulin and/or sulfonylurea therapy; $24.6 \%$ of patients were using metformin at screening and underwent the metformin wash-off. Approximately half of the patients had a history of cardiovascular disease or heart failure.

Baseline characteristics were similar in the overall cohort and the subgroup that excluded patients who were identified as using prohibited metformin after randomization (proportion of males: $49.5 \%$ and 50.1\%; mean baseline A1C: $8.2 \%$ and $8.1 \%$; mean eGFR: $46.6 \mathrm{~mL} / \mathrm{min} /$ $1.73 \mathrm{~m}^{2}$ and $46.1 \mathrm{~mL} / \mathrm{min} / 1.73 \mathrm{~m}^{2}$; proportion on metformin at screening: $24.6 \%$ and $22.4 \%$, respectively). Likewise, baseline characteristics in the stage 3A CKD cohort and its subgroup excluding patients who tested positive for metformin use were similar (proportion of males: 49.8\% and 51.4\%; mean baseline A1C: $8.2 \%$ and $8.1 \%$; mean eGFR: 50.9 and $50.7 \mathrm{~mL} / \mathrm{min} /$ $1.73 \mathrm{~m}^{2}$; proportion on metformin at screening: $27.6 \%$ and $25.5 \%$, respectively).

\section{Efficacy}

A1C

Ertugliflozin $5 \mathrm{mg}$ and $15 \mathrm{mg}$ resulted in reductions from baseline in $\mathrm{A} 1 \mathrm{C}$ in the overall and stage 3A CKD cohorts (Fig. 1a, b, Table 2). 
Table 1 Baseline demographics and disease characteristics (overall cohort)

\begin{tabular}{|c|c|c|c|c|}
\hline & $\begin{array}{l}\text { Placebo } \\
(n=154)\end{array}$ & $\begin{array}{l}\text { Ertugliflozin } 5 \mathrm{mg} \\
(n=158)\end{array}$ & $\begin{array}{l}\text { Ertugliflozin } 15 \mathrm{mg} \\
(n=155)\end{array}$ & $\begin{array}{l}\text { Total } \\
(n=467)\end{array}$ \\
\hline Age, years & $67.5(8.9)$ & $66.7(8.3)$ & $67.5(8.5)$ & $67.3(8.6)$ \\
\hline Male, $n(\%)$ & $72(46.8)$ & $84(53.2)$ & $75(48.4)$ & $231(49.5)$ \\
\hline \multicolumn{5}{|l|}{ Race, $n(\%)$} \\
\hline White & $134(87.0)$ & $127(80.4)$ & $119(76.8)$ & $380(81.4)$ \\
\hline Asian & $9(5.8)$ & $16(10.1)$ & $20(12.9)$ & $45(9.6)$ \\
\hline Black or African American & $4(2.6)$ & $6(3.8)$ & $9(5.8)$ & $19(4.1)$ \\
\hline American Indian or Alaska Native & $1(0.6)$ & $0(0)$ & $0(0)$ & $1(0.2)$ \\
\hline Multiple & $6(3.9)$ & $9(5.7)$ & $7(4.5)$ & $22(4.7)$ \\
\hline \multicolumn{5}{|l|}{ Ethnicity, $n$ (\%) } \\
\hline Hispanic or Latino & $27(17.5)$ & $29(18.4)$ & $31(20.0)$ & $87(18.6)$ \\
\hline \multicolumn{5}{|l|}{ Region, $n(\%)$} \\
\hline North America & $41(26.6)$ & $55(34.8)$ & $38(24.5)$ & $134(28.7)$ \\
\hline South America & $17(11.0)$ & $17(10.8)$ & $20(12.9)$ & $54(11.6)$ \\
\hline Europe & $70(45.5)$ & $54(34.2)$ & $62(40.0)$ & $186(39.8)$ \\
\hline Asia & $23(14.9)$ & $24(15.2)$ & $33(21.3)$ & $80(17.1)$ \\
\hline South Africa & $3(1.9)$ & $8(5.1)$ & $2(1.3)$ & $13(2.8)$ \\
\hline Duration of T2DM, years & $13.1(8.1)$ & $14.9(9.0)$ & $14.5(8.5)$ & $14.2(8.5)$ \\
\hline Body weight, kg & $90.4(18.9)$ & $89.4(22.5)$ & $85.8(17.4)$ & $88.5(19.8)$ \\
\hline BMI, $\mathrm{kg} / \mathrm{m}^{2}$ & $33.2(6.1)$ & $32.6(6.8)$ & $31.7(5.3)$ & $32.5(6.1)$ \\
\hline $\mathrm{AlC}, \%$ & $8.1(0.9)$ & $8.2(1.0)$ & $8.2(0.9)$ & $8.2(0.9)$ \\
\hline $\mathrm{FPG}, \mathrm{mg} / \mathrm{dL}$ & $156.9(56.4)$ & $160.9(56.4)$ & $157.5(47.8)$ & $158.5(53.6)$ \\
\hline $\mathrm{eGFR}, \mathrm{mL} / \mathrm{min} / 1.73 \mathrm{~m}^{2}$ & $46.0(9.4)$ & $46.8(7.8)$ & $46.9(9.1)$ & $46.6(8.8)$ \\
\hline $\begin{array}{l}\text { Medical history of CV disease or heart } \\
\text { failure, } n(\%)\end{array}$ & $76(49.4)$ & $79(50.0)$ & $77(49.7)$ & $232(49.7)$ \\
\hline \multicolumn{5}{|c|}{ Background AHA therapy at screening, $n$ (\%) } \\
\hline Currently on AHA therapy & $151(98.1)$ & $153(96.8)$ & $148(95.5)$ & $452(96.8)$ \\
\hline Biguanides & $36(23.4)$ & $41(25.9)$ & $38(24.5)$ & $115(24.6)$ \\
\hline DPP-4 inhibitors & $21(13.6)$ & $22(13.9)$ & $20(12.9)$ & $63(13.5)$ \\
\hline GLP-1 receptor agonists & $7(4.5)$ & $3(1.9)$ & $3(1.9)$ & $13(2.8)$ \\
\hline Insulins and analogs & $85(55.2)$ & $89(56.3)$ & $87(56.1)$ & $261(55.9)$ \\
\hline Sulfonylureas & $63(40.9)$ & $65(41.1)$ & $60(38.7)$ & $188(40.3)$ \\
\hline Others & $8(5.2)$ & $8(5.1)$ & $9(5.8)$ & $25(5.4)$ \\
\hline
\end{tabular}

Data are mean $( \pm S D)$ unless otherwise stated

$A H A$ antihyperglycemic agents, $A I C$ glycated hemoglobin, $B M I$ body mass index, $C V$ cardiovascular, $D P P-4$ dipeptidyl peptidase 4, eGFR estimated glomerular filtration rate, $F P G$ fasting plasma glucose, GLP-1 glucagon-like peptide 1, $S D$ standard deviation, $T 2 D M$ type 2 diabetes mellitus 

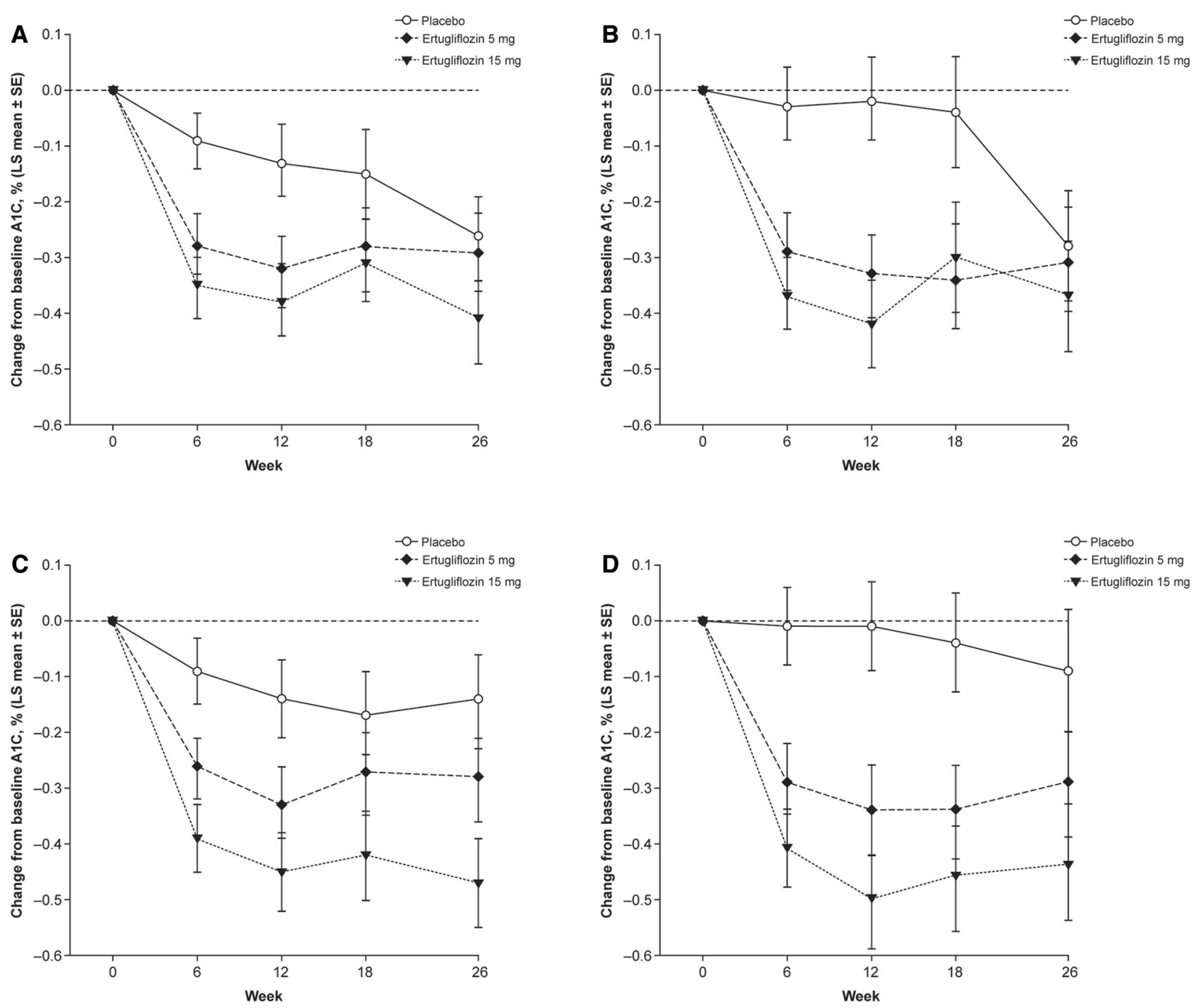

Fig. 1a-d Changes in glycated hemoglobin over time in: a the overall cohort [estimated glomerular filtration rate (eGFR) $\geq 30$ to $<60 \mathrm{~mL} / \mathrm{min} / 1.73 \mathrm{~m}^{2}$ ]; b the stage $3 \mathrm{~A}$ chronic kidney disease (CKD) cohort (eGFR $\geq 45$

to $<60 \mathrm{~mL} / \mathrm{min} / 1.73 \mathrm{~m}^{2}$ ); $\mathrm{c}$ the overall cohort excluding metformin users (post hoc analysis); $\mathbf{d}$ the stage $3 \mathrm{~A}$ CKD cohort excluding metformin users (post hoc analysis). LS least squares, $S E$ standard error

An unusual placebo response was observed, characterized by notable decreases in $\mathrm{A} 1 \mathrm{C}$ between week 18 and week 26 . The large placebo response resulted in nonsignificant differences in the $\mathrm{A} 1 \mathrm{C}$ change from baseline between the placebo and the ertugliflozin groups at week 26; as such, hypothesis testing stopped after the first test. After week 26, the placebo and ertugliflozin responses were generally stable through week 52 (Fig. S3 in the ESM). Reported compliance with ertugliflozin was high in phase $\mathrm{A}+\mathrm{B}$, with $98.7 \%$ and $99.4 \%$ of

patients being $\geq 75 \%$ compliant with the study treatment in the ertugliflozin $5 \mathrm{mg}$ and $15 \mathrm{mg}$ groups, respectively.

Post-hoc analyses (described in the "Methods" section) were conducted to determine whether the placebo response could have been the result of the surreptitious use of metformin [21]. The proportions of patients with plasma samples available for metformin evaluation were similar across treatment groups and eGFR strata at each visit (week 6: 74.5-91.3\%; week 12: 83.7-94.3\%; week 18: 88.7-94.3\%; week 26: 
Table 2 Glycated hemoglobin change from baseline at week 26 and week 52

\begin{tabular}{|c|c|c|c|}
\hline & \multicolumn{3}{|l|}{ Week 26} \\
\hline & Placebo & Ertugliflozin $5 \mathrm{mg}$ & Ertugliflozin $15 \mathrm{mg}$ \\
\hline \multicolumn{4}{|l|}{ Overall cohort (primary analysis) } \\
\hline & $n=154$ & $n=158$ & $n=155$ \\
\hline LS mean $(95 \% \mathrm{CI})$ & $-0.3(-0.4,-0.1)$ & $-0.3(-0.4,-0.1)$ & $-0.4(-0.6,-0.3)$ \\
\hline Difference in LS means (95\% CI) versus placebo & - & $-0.0(-0.2,0.2)$ & $-0.2(-0.4,0.1)$ \\
\hline$P$ value & - & $0.807^{\mathrm{b}}$ & 0.155 \\
\hline \multicolumn{4}{|l|}{ Stage 3A CKD cohort (secondary analysis) } \\
\hline & $n=99$ & $n=105$ & $n=97$ \\
\hline LS mean $(95 \% \mathrm{CI})$ & $-0.3(-0.5,-0.1)$ & $-0.3(-0.5,-0.1)$ & $-0.4(-0.6,-0.2)$ \\
\hline Difference in LS means ( $95 \% \mathrm{CI}$ ) versus placebo & - & $-0.0(-0.3,0.2)$ & $-0.1(-0.4,0.2)$ \\
\hline$P$ value $^{\mathrm{b}}$ & - & 0.828 & 0.496 \\
\hline \multicolumn{4}{|c|}{ Overall cohort (post hoc analysis excluding metformin users) } \\
\hline & $n=128$ & $n=134$ & $n=127$ \\
\hline LS mean $(95 \% \mathrm{CI})$ & $-0.1(-0.3,0.0)$ & $-0.3(-0.4,-0.1)$ & $-0.5(-0.6,-0.3)$ \\
\hline Difference in LS means (95\% CI) versus placebo & - & $-0.1(-0.4,0.1)$ & $-0.3(-0.6,-0.1)$ \\
\hline \multicolumn{4}{|c|}{ Stage 3A CKD cohort (post hoc analysis excluding metformin users) } \\
\hline & $n=79$ & $n=89$ & $n=75$ \\
\hline LS mean $(95 \% \mathrm{CI})$ & $-0.1(-0.3,0.1)$ & $-0.3(-0.5,-0.1)$ & $-0.4(-0.7,-0.2)$ \\
\hline \multirow[t]{3}{*}{ Difference in LS means ( $95 \% \mathrm{CI}$ ) versus placebo } & - & $-0.2(-0.5,0.1)$ & $-0.4(-0.6,-0.1)$ \\
\hline & \multicolumn{3}{|l|}{ Week 52 } \\
\hline & Placebo & $\begin{array}{l}\text { Ertugliflozin } \\
5 \mathrm{mg}\end{array}$ & $\begin{array}{l}\text { Ertugliflozin } \\
15 \mathrm{mg}\end{array}$ \\
\hline \multicolumn{4}{|l|}{ Stage 3A CKD cohort (secondary analysis) } \\
\hline & $n=99$ & $n=105$ & $n=97$ \\
\hline LS mean $(95 \% \mathrm{CI})$ & $-0.3(-0.5,-0.0)$ & $-0.2(-0.5,-0.0)$ & $-0.4(-0.6,-0.2)$ \\
\hline Difference in LS means ( $95 \% \mathrm{CI}$ ) versus placebo & - & $0.0(-0.3,0.3)$ & $-0.1(-0.4,0.2)$ \\
\hline \multicolumn{4}{|c|}{ Stage 3A CKD cohort (post hoc analysis excluding metformin users) } \\
\hline & $n=79$ & $n=89$ & $n=75$ \\
\hline LS mean $(95 \% \mathrm{CI})$ & $0.0(-0.3,0.3)$ & $-0.2(-0.5,0.1)$ & $-0.4(-0.6,-0.1)$ \\
\hline Difference in LS means ( $95 \% \mathrm{CI}$ ) versus placebo & - & $-0.2(-0.6,0.2)$ & $-0.4(-0.8,0.0)$ \\
\hline
\end{tabular}

$C K D$ chronic kidney disease, $C I$ confidence interval, $L S$ least squares, $N D$ not determined (i.e., not pre-specified per protocol)

a Analyses of the overall cohort were not planned for week 52

b As the primary hypothesis test was not statistically significant, the multiplicity strategy did not permit the testing of any further hypotheses, and $P$ values are provided for descriptive purposes only 
59.2-76.3\%); week 26 samples were available only for patients who agreed to provide a biomarker sample, and therefore fewer patients had metformin assays for that time point. The percentages of patients with positive metformin assay results were similar across the three treatment groups (Table S2 in the ESM).

In the post hoc analysis examining the treatment response in patients who did not test positive for metformin, the placebo-corrected least squares (LS) mean differences in A1C at week 26 for ertugliflozin 5 and $15 \mathrm{mg}$ in the overall cohort were $-0.1 \%(95 \% \mathrm{CI}-0.4,0.1)$ and $-0.3 \% \quad(95 \%$ CI $-0.6,-0.1) \quad$ (Table 2$)$. Excluding data from patients with positive metformin tests had little impact on the magnitude of the A1C lowering in the ertugliflozin groups, but resulted in smaller reductions in $\mathrm{A} 1 \mathrm{C}$ in the placebo group (Fig. 1c, d). Given that the metformin use was not reported, the doses of metformin taken and the frequency and duration of dosing are not known. Although metformin use was detected in all treatment groups, among metformin users, LS mean reductions from baseline in $\mathrm{A} 1 \mathrm{C}$ were larger in the placebo group $(0.7 \%)$ compared with the ertugliflozin groups $(0.3 \%$ and $0.1 \%$ for ertugliflozin 5 and $15 \mathrm{mg}$, respectively), which may suggest more intensive and/or frequent use of metformin in the placebo group.

In the stage $3 \mathrm{~A}$ CKD cohort, the placebo-adjusted LS mean changes from baseline in $\mathrm{A} 1 \mathrm{C}$ at week 26 were $-0.2 \%$ (95\% CI $-0.5,0.1)$ and $-0.4 \%(95 \% \mathrm{CI}-0.6,-0.1)$ in the ertugliflozin $5 \mathrm{mg}$ and $15 \mathrm{mg}$ groups, respectively (post hoc analysis excluding patients who tested positive for metformin use; Table 2).

The LS mean changes from baseline in A1C at week 26 in the stage 3B CKD cohort (including metformin users) were $-0.2 \%,-0.3 \%$, and $-0.5 \%$ in the placebo, ertugliflozin $5 \mathrm{mg}$, and ertugliflozin $15 \mathrm{mg}$ groups, respectively; however, in contrast with the overall and stage $3 \mathrm{~A}$ CKD cohorts, there was no early or sustained separation of the ertugliflozin and placebo groups (data not shown). Metformin use was less common in the stage 3B CKD cohort (10.3-15.1\% of patients with at least one sample positive for metformin across the treatment groups) compared to the stage 3A CKD cohort (15.2-22.7\%).

In a pre-specified analysis conducted in the stage 3A CKD cohort (and including metformin users), the odds of having an $\mathrm{A} 1 \mathrm{C}<7.0 \%$ at week 26 were similar in the ertugliflozin and placebo groups (Table 3 ).

\section{Other Efficacy Endpoints}

These endpoints were pre-specified for analysis in the stage $3 \mathrm{~A} \mathrm{CKD}$ cohort, and the analyses included data from patients who tested positive for metformin use. Relative to placebo, ertugliflozin led to greater reductions from baseline in FPG at week 26 (Table 3). A post hoc analysis excluding data from metformin users yielded similar results to the pre-specified analysis (data not shown).

The LS mean reductions from baseline in body weight and SBP at week 26 were greater in the ertugliflozin groups than in the placebo group (Table 3). The changes in FPG, body weight, and SBP at week 52 are shown in Table 3 and Fig. S4 of the ESM.

\section{Safety}

The primary analysis of safety was performed in the overall cohort including all data up to week 26; safety was also analyzed in this cohort including all data up to week 52 .

At weeks 26 and 52, the overall incidence of AEs and of SAEs was generally similar across groups (Table 4). The observed incidence of drug-related AEs was highest in the ertugliflozin $5 \mathrm{mg}$ group at both 26 and 52 weeks and was partly due to an observed higher incidence of hypoglycemia AEs. This analysis included patients that required rescue therapy. Compared with placebo, more patients discontinued due to an $\mathrm{AE}$ in the ertugliflozin $5 \mathrm{mg}$ group, but not in the ertugliflozin $15 \mathrm{mg}$ group; there was no specific AE or group of AEs responsible for this. Deaths were balanced across the treatment groups, with $3(1.9 \%), 3(1.9 \%)$, and 4 $(2.6 \%)$, respectively, for the placebo, ertugliflozin $5 \mathrm{mg}$, and ertugliflozin $15 \mathrm{mg}$ groups. One additional death was reported in the 


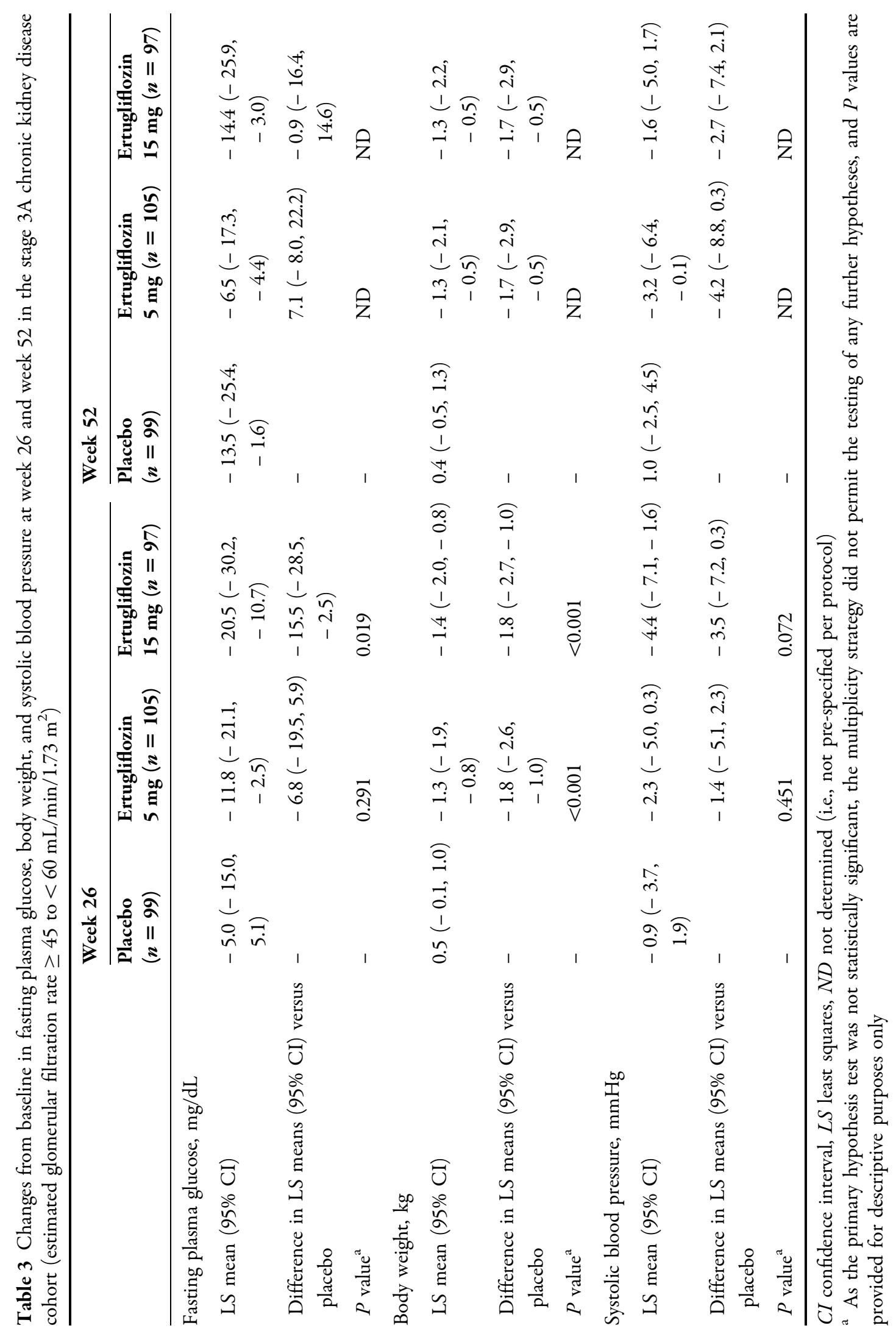




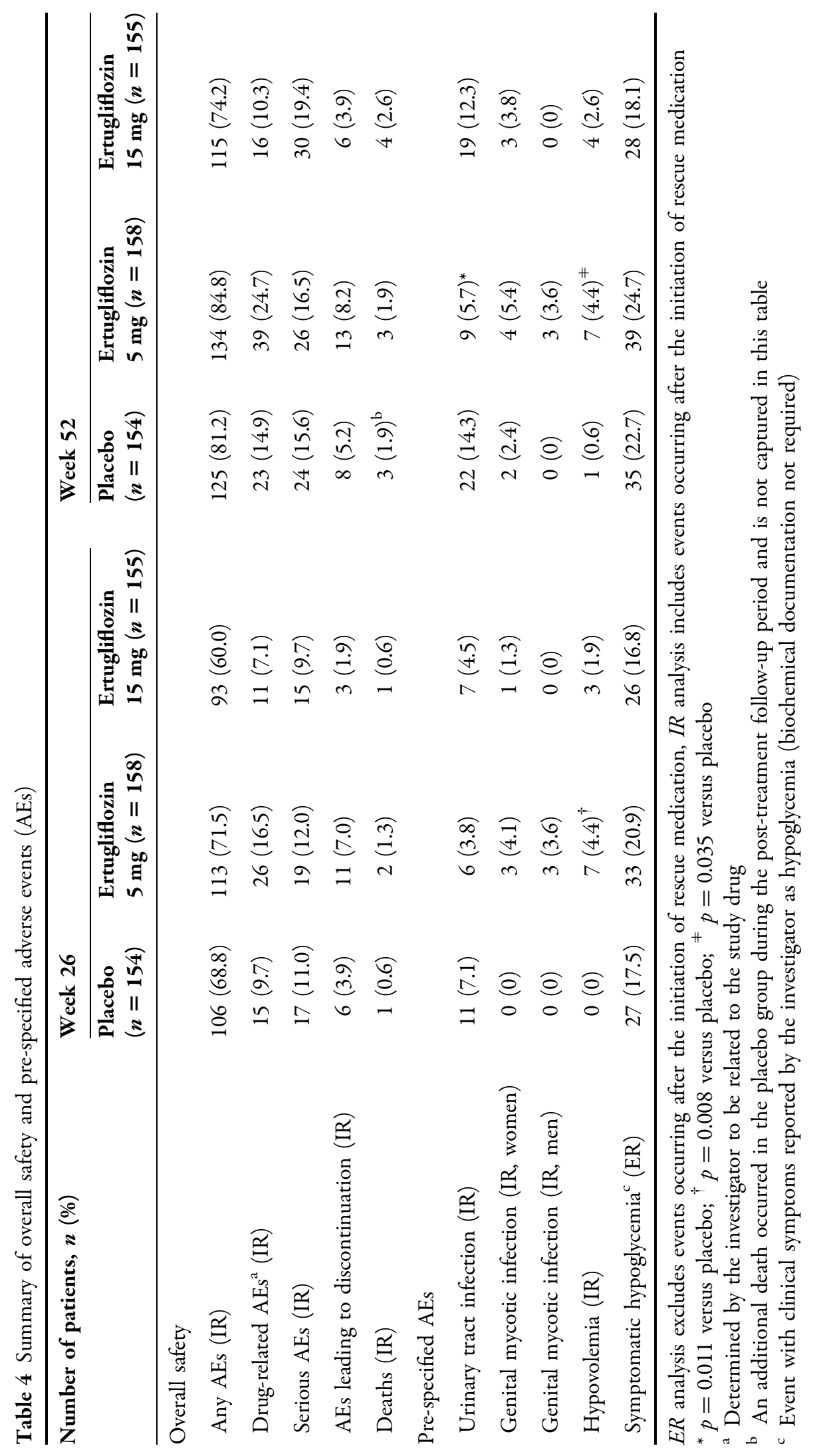


placebo group during the post-treatment follow-up period.

In the overall cohort, the incidence of symptomatic hypoglycemia was similar across the treatment groups at weeks 26 and 52 (Table 4). In the stage 3A CKD cohort, the observed incidence of symptomatic hypoglycemia was lower in the ertugliflozin $15 \mathrm{mg}$ group (12.4\%) compared with the placebo (21.2\%) and ertugliflozin $5 \mathrm{mg}(23.8 \%)$ groups. The incidence of symptomatic hypoglycemia at week 52 was similar across treatment groups in the stage 3B CKD cohort $(25.5 \%, 26.4 \%$ and $27.6 \%$ for placebo, ertugliflozin $5 \mathrm{mg}$, and ertugliflozin $15 \mathrm{mg}$, respectively).

The incidence of documented hypoglycemia at week 26 was $33.1 \%, 34.2 \%$, and $25.2 \%$ in the placebo, ertugliflozin $5 \mathrm{mg}$, and ertugliflozin $15 \mathrm{mg}$ groups, respectively, and 39.6\%, 39.9\%, and $27.7 \%$, respectively, at week 52 . The total number of documented hypoglycemia episodes was greater in the ertugliflozin groups than in the placebo group (placebo, 187 and 293; ertugliflozin $5 \mathrm{mg}$ : 358 and 526; ertugliflozin $15 \mathrm{mg}$ : 213 and 376 at weeks 26 and 52, respectively). Results of post hoc analyses of documented hypoglycemia excluding metformin users were consistent with results from the overall cohort, indicating that surreptitious metformin use did not meaningfully impact the incidence of hypoglycemia (data not shown).

At weeks 26 and 52, the incidence of urinary tract infection was not higher in the ertugliflozin groups compared to the placebo group, and the incidence of GMI AEs was similar in the ertugliflozin groups and the placebo group. The incidence of hypovolemia AEs was significantly higher in the ertugliflozin $5 \mathrm{mg}$ group compared to placebo, and higher in the ertugliflozin $15 \mathrm{mg}$ group versus placebo at weeks 26 and 52 . Of the 11 ertugliflozin-treated patients (ertugliflozin $5 \mathrm{mg}, n=7$; ertugliflozin $15 \mathrm{mg}$, $n=4$ ) who experienced a hypovolemia AE by week $52,18.2 \%$ were $\geq 75$ years of age (vs $21.6 \%$ of study participants overall), $81.8 \%$ were taking diuretics (vs $50.5 \%$ in the study overall), and $72.7 \%$ were in the stage 3A CKD cohort (vs 66\% of patients in the study overall).

Modest reductions from baseline in mean eGFR were observed in the ertugliflozin groups

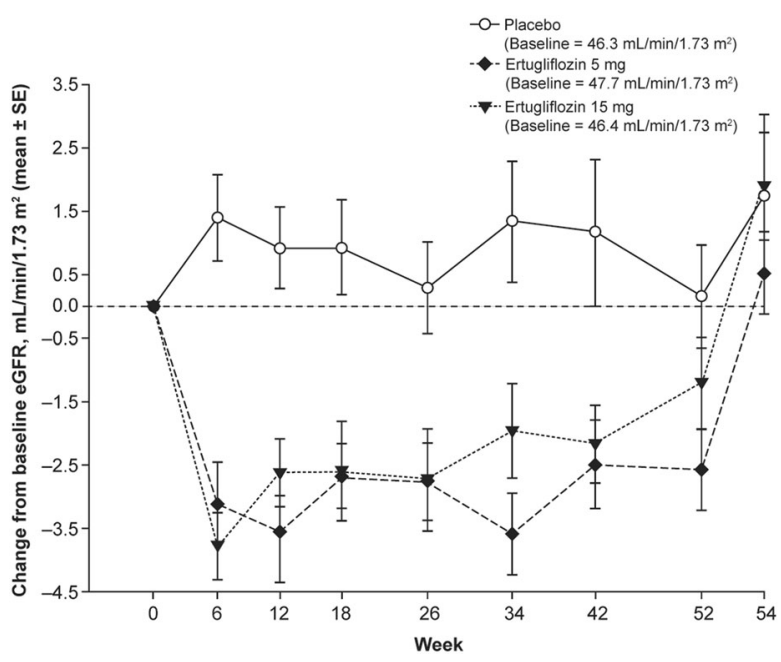

Fig. 2 Mean change from baseline in estimated glomerular filtration rate $(\mathrm{eGFR})\left(\mathrm{mL} / \mathrm{min} / 1.73 \mathrm{~m}^{2}\right)$ through week 54. SE standard error

at week 6 (first post-randomization visit), and were followed by a slight increase toward baseline, but they remained below baseline through week 52 (Fig. 2). The magnitude of the week 6 change from baseline was similar for the stage $3 \mathrm{~A}$ and $3 \mathrm{~B}$ CKD cohorts (data not shown). Analysis of the post-treatment eGFR change from baseline showed that eGFR returned to baseline levels within 2 weeks of study drug cessation (Fig. 2). Similar findings were seen in a complementary analysis including patients who discontinued study medication early. In categorical analyses of the overall cohort, the proportions of patients who had any occurrence of a decrease $>30 \%$ from baseline in eGFR at weeks 26 and 52 were higher in the ertugliflozin $5 \mathrm{mg}$ and ertugliflozin $15 \mathrm{mg}$ groups (week 26: $10.3 \%$ and $8.7 \%$, respectively; week 52 : $13.5 \%$ and $14.0 \%$, respectively) than in the placebo group (week 26: 2.6\%; week 52: 7.3\%). No patients in the ertugliflozin $5 \mathrm{mg}$ group, one $(0.7 \%)$ in the ertugliflozin $15 \mathrm{mg}$ group, and two $(1.3 \%)$ in the placebo group had at least one eGFR decrease $>50 \%$. Renal-related AEs (defined according to a standard MedDRA composite query of acute kidney injury, acute prerenal failure, or renal impairment) were reported in $0.6 \%, 2.5 \%$, and $1.3 \%$ of patients in the placebo, ertugliflozin $5 \mathrm{mg}$, and 
ertugliflozin $15 \mathrm{mg}$ groups, respectively, at week 26 , and in $1.3 \%, 3.2 \%$, and $1.3 \%$ of patients, respectively, at week 52 .

Approximately $44-47 \%$ of patients were normoalbuminuric at baseline across the treatment groups; however, fewer patients were microalbuminuric and more were macroalbuminuric in the ertugliflozin groups relative to the placebo group. At week 52, a similar proportion of patients had changed from normoalbuminuric at baseline to microalbuminuric across treatment groups; none changed from normoalbuminuric at baseline to macroalbuminuric at week 52 .

No notable between-group differences in the occurrence of adverse hyperkalemia events or blood potassium increase events that meet PDLC criteria for potassium were observed in the overall cohort.

The placebo-adjusted difference in LS mean percent change from baseline in LDL-C at week 26 was $6.4 \%$ and $10.4 \%$ in the ertugliflozin $5 \mathrm{mg}$ and ertugliflozin $15 \mathrm{mg}$ groups and $9.1 \%$ and $6.6 \%$, respectively, at week 52. No notable changes in HDL-C were seen after 26 weeks (placebo-adjusted difference in LS mean percent changes from baseline at week 26 : $-0.7 \%$ and $-0.7 \%$ for ertugliflozin $5 \mathrm{mg}$ and ertugliflozin $15 \mathrm{mg}$, respectively). After 52 weeks, the placebo-adjusted mean percent increase from baseline in HDL-C were $2.2 \%$ and $5.7 \%$ in the ertugliflozin $5 \mathrm{mg}$ and ertugliflozin $15 \mathrm{mg}$ groups, respectively.

In the ertugliflozin groups, small mean increases from baseline in hemoglobin were seen from week 6 (ertugliflozin $5 \mathrm{mg}, 0.4 \mathrm{~g} / \mathrm{dL}$; ertugliflozin $15 \mathrm{mg}, 0.4 \mathrm{~g} / \mathrm{dL}$ ) through week 52 (ertugliflozin $5 \mathrm{mg}, \quad 0.8 \mathrm{~g} / \mathrm{dL}$; ertugliflozin $15 \mathrm{mg}, 0.9 \mathrm{~g} / \mathrm{dL}$ ); no meaningful change over time was observed in the placebo group. Relative to placebo, higher proportions of patients in the ertugliflozin groups met the PDLC criterion of an increase in hemoglobin $>2.0 \mathrm{mg} / \mathrm{dL}$ ( $\geq 1$ occurrence) from baseline through week 26 (ertugliflozin $5 \mathrm{mg}, 9.0 \%$; ertugliflozin $15 \mathrm{mg}$, 8.1\%; placebo, $2.0 \%$ ) and through week 52 (ertugliflozin $5 \mathrm{mg}, 14.7 \%$; ertugliflozin $15 \mathrm{mg}$, 19.5\%; placebo, $2.6 \%$ ).

Small mean increases in magnesium and phosphate were observed from week 6 through week 26 in the ertugliflozin groups, while no meaningful changes over time were observed in the placebo group. No notable changes over time in calcium levels were observed across the three treatment groups.

At week 26, a higher proportion of patients in the ertugliflozin $5 \mathrm{mg}$ group had at least one parathyroid hormone (PTH) value that met PDLC criteria [PTH increase $\geq 20 \%$ and value $>$ upper limit of normal (ULN) or PTH increase $\geq 30 \%$ ] relative to placebo; the proportions were similar in the ertugliflozin $15 \mathrm{mg}$ and placebo groups. The proportions of patients meeting PDLC criteria for PTH were similar across the groups at week 52 .

At week 52, four patients in the ertugliflozin $5 \mathrm{mg}$ group and one patient in the placebo group had a confirmed fracture. These were all due to a fall; none were associated with hypoglycemia- or hypovolemia-related events.

\section{DISCUSSION}

In this phase 3 randomized study conducted in patients with T2DM and stage 3 CKD (VERTIS RENAL), ertugliflozin provided reductions in A1C after 26 weeks that were not statistically significant compared with placebo. However, metformin, which was used by approximately $17 \%$ of patients, modified the primary endpoint response. Metformin use was not reported to investigators and was discovered after phase A by analysis of retained plasma samples, and contrasts with the use of rescue medication, which was reported to investigators. It is possible that patients self-medicated based on fingerstick glucose measurements, which were performed by patients during the study to monitor glucose levels, which is required for safety monitoring (for hypo- and hyperglycemia) and assessing glycemic rescue requirements. A small number $(6 \%)$ of patients initiated the allowed non-metformin glycemic rescue therapy during the trial; this proportion was roughly similar across treatment groups.

At the time of initiation of this global study, metformin was not recommended for use in patients with moderate renal impairment, and it was therefore a prohibited medication. 
Consequently, patients who were taking metformin at the time of screening $(\sim 25 \%$ of patients) were required to wash off metformin prior to randomization. These patients accounted for $41 \%$ of the 78 patients who tested positive for metformin use post-randomization. Metformin is available without a prescription in the Russian Federation and Romania [21]; sites in those countries accounted for $28 \%$ of the metformin users. Overall, many patients had access to metformin and had the ability to use metformin without the knowledge of the investigators. Additionally, patients enrolled in the study were supplied with glucose meters and test strips and counseled to measure their glucose levels throughout the course of the study (at least twice weekly, or more frequently if required by the investigator). Thus, it is possible that some patients self-medicated with metformin in response to fingerstick glucose measurements. This might also explain why the reduction in $\mathrm{A} 1 \mathrm{C}$ was greater in metformin users allocated to placebo than to ertugliflozin, despite the fact that similar proportions of patients used metformin across treatment arms (i.e., patients may have used metformin more frequently or taken higher doses in the placebo group in response to fingerstick glucose measurements). A greater response to metformin might also be expected in those using placebo compared to those on ertugliflozin, since the use of an active agent might partially attenuate the effect of a co-administered AHA. After the initiation of the study, the U.S. Food and Drug Administration concluded that metformin can be used in some patients with moderate renal impairment, and has mandated changes to the labeling of all metformin-containing medicines to reflect this [22].

A large placebo response was also observed in a study of another SGLT2 inhibitor, dapagliflozin, in patients with moderate renal impairment [5]. Unlike in our study, the dapagliflozin study showed no early separation in the change in $\mathrm{A} 1 \mathrm{C}$ over time curves for the placebo and drug treatment arms. In our study, the percentage of patients who discontinued study medication or discontinued the study was similar among the treatment arms (Fig. S2 in the ESM), making differential dropout an unlikely source of the observed placebo response. Patients enrolled in the present study were meant to keep their background AHA treatment regimen stable; however, those requiring intensification or meeting hyperglycemic rescue criteria were to titrate existing agents or add new agents. All such post-rescue data were censored in the presented A1C analyses, which also suggests that the observed placebo response was likely not the result of the intensification of the AHA regimen that was reported to investigators. We note that the percentage of patients meeting the hyperglycemic rescue criteria through week 52 was similar among the treatment arms (32.0-36.4\%), and a Kaplan-Meier analysis of time to glycemic rescue did not reveal any differences between treatment arms. The analysis of glycemic efficacy may have been affected by metformin use, so the post hoc analysis excluding data from patients who were known to have used metformin likely provides a better estimate of the effects of ertugliflozin on A1C than the primary analysis. Fewer patients had metformin assays available at week 26 than at previous time points, so it is consequently possible that the number of metformin users at week 26 has been underestimated. Nevertheless, this post hoc analysis suggests that ertugliflozin $15 \mathrm{mg}$ lowers $\mathrm{A} 1 \mathrm{C}$ in patients with stage $3 \mathrm{CKD}$ versus placebo. The degree of A1C lowering from baseline with ertugliflozin was almost identical to that observed with other SGLT2 inhibitors in this patient population (dapagliflozin $5 \mathrm{mg}$ and $10 \mathrm{mg}$ at week 24 : $-0.4 \%$ and $-0.4 \%$, respectively, in patients with eGFR $\geq 30$ and $<60 \mathrm{~mL} / \mathrm{min} / 1.73 \mathrm{~m}^{2}$; canagliflozin $100 \mathrm{mg}$ and $300 \mathrm{mg}$ at week 24 : $-0.3 \%$ and $-0.4 \%$, respectively, in patients with eGFR $\geq 30$ and $<50 \mathrm{~mL} / \mathrm{min} / 1.73 \mathrm{~m}^{2}$; empagliflozin $25 \mathrm{mg}$ at week $24:-0.4 \%$ in patients with eGFR $\geq 30$ and $<60 \mathrm{~mL} / \mathrm{min} / 1.73 \mathrm{~m}^{2}$ ) [5-7].

The efficacy of ertugliflozin in patients with stage $3 \mathrm{CKD}$ is supported by additional observations in the pre-specified analysis (i.e., including metformin users). For example, the analysis of body weight or BP would not be expected to be influenced by metformin use. Greater reductions in body weight were observed for ertugliflozin $5 \mathrm{mg}$ and $15 \mathrm{mg}$ versus placebo $(p<0.001$ for both comparisons). 
There were also small reductions in SBP for ertugliflozin $5 \mathrm{mg}$ and $15 \mathrm{mg}$ versus placebo. These additional benefits in relation to body weight and BP are similar to those observed with empagliflozin in patients with moderate renal impairment [6]. Additional evidence of ertugliflozin efficacy in this patient population is provided by the observed early complete separation of the ertugliflozin and placebo groups for A1C change from baseline and the greater reduction in FPG for ertugliflozin $15 \mathrm{mg}$ versus placebo $(p=0.019)$. Analyses of the efficacy endpoints at week 52 showed that the effects of ertugliflozin on A1C and body weight were maintained over 52 weeks of treatment.

In a phase 1 pharmacokinetics and pharmacodynamics study, administration of a single dose of ertugliflozin $15 \mathrm{mg}$ to patients with T2DM and stage 3 CKD (eGFR $\geq 30$ and $\leq 59 \mathrm{~mL} / \mathrm{min} / 1.73 \mathrm{~m}^{2}$ ) resulted in substantial UGE (observed median $\mathrm{UGE}_{0-24}$ of $33.4 \mathrm{~g}$ ), which was approximately $48 \%$ of the median value in T2DM patients with eGFR $\geq 90 \mathrm{~mL} /$ $\min / 1.73 \mathrm{~m}^{2}$ (observed median $\mathrm{UGE}_{0-24}$ of $69.3 \mathrm{~g}$ ) noted in the same study [23]. A regression-model-predicted mean $\mathrm{UGE}_{0-24}$ for a patient with an eGFR of $52.5 \mathrm{~mL} / \mathrm{min} / 1.73 \mathrm{~m}^{2}$ (midpoint of stage 3A CKD) is $29.5 \mathrm{~g}$. Given that SGLT2 inhibitors reduce A1C by promoting UGE, these data are direct evidence of the pharmacodynamic activity of ertugliflozin in patients with T2DM and stage 3 CKD. The relative reductions in UGE in patients with moderate renal impairment compared to patients with normal renal function for ertugliflozin are similar in magnitude (approximately 50\%) to those reported for other SGLT2 inhibitors [24-26].

Both doses of ertugliflozin had an acceptable safety profile, with no clinically meaningful differences in the overall incidences of AEs, SAEs, or AEs leading to discontinuation across groups. In contrast to the evaluation of glycemic efficacy, the analysis of safety is not expected to be affected by metformin use, with the potential exception of hypoglycemia. In this study, approximately $96 \%$ of patients were using insulin and/or a sulfonylurea, consistent with the overall high incidence of hypoglycemia that was observed across the three groups. The incidence of symptomatic and documented hypoglycemia was similar in each ertugliflozin group compared with the placebo group. There were no clinically meaningful differences in the incidence of GMIs. SGLT2 inhibitors are generally associated with an increased incidence of GMIs, and while this association was observed with ertugliflozin in patients without moderate renal impairment [27], both a lower overall incidence of GMIs and no increase in risk relative to placebo were observed in patients with moderate renal impairment in the present study. This result was inconsistently obtained in other studies of SGLT2 inhibitors in this population, with some studies showing an increased risk $[5,28]$ and another not [6]. The incidence of renal-function-related AEs was low overall and more frequent in the ertugliflozin groups than in the placebo group. The observation of hypovolemia-related AEs and decreases in eGFR are also evidence of the pharmacological activity of ertugliflozin in patients with stage 3 CKD. Most patients experiencing hypovolemia were taking a diuretic medication. Decreases from baseline to week 6 in eGFR were followed by a slight increase, but it remained below baseline through week 52 . The reversible changes in eGFR observed upon cessation of ertugliflozin treatment in this study further support the pharmacodynamic activity of ertugliflozin in this patient population and the hypothesis that eGFR reductions observed with SGLT2 inhibitors are hemodynamically mediated and are not the result of renal injury $[4,6]$.

\section{CONCLUSIONS}

In patients with T2DM and stage 3 CKD who were inadequately controlled on their existing antihyperglycemic therapy, the addition of ertugliflozin showed evidence of pharmacologic activity in glycemic control and body weight and $\mathrm{BP}$ reduction, although the comparison with the placebo group was not statistically significant. Ertugliflozin was generally well tolerated and the safety profile was largely consistent with that observed in phase 3 clinical trials of patients with T2DM and normal renal 
function, except for a higher incidence of hypovolemia in the present study.

\section{ACKNOWLEDGEMENTS}

The authors wish to thank the investigators, staff, and participants in the VERTIS RENAL trial (protocol MK-8835-001).

Merck Sharp \& Dohme Corp., a subsidiary of Merck \& Co., Inc., Kenilworth, NJ, USA, in collaboration with Pfizer Inc, provided financial support for this study. Merck Sharp \& Dohme Corp., a subsidiary of Merck \& Co, Inc., Kenilworth, NJ, USA, provided funding for article processing charges for this publication. All authors had full access to all of the data in this study and take complete responsibility for the integrity of the data and accuracy of the data analysis.

All named authors meet the International Committee of Medical Journal Editors (ICMJE) criteria for authorship for this manuscript, take responsibility for the integrity of the work as a whole, and have given final approval for the version to be published.

Medical writing support, including assisting authors with the development of the outline and initial draft, was provided by Camille Bonomelli, $\mathrm{PhD}$, and comments were incorporated by Elien Moës, PhD, ISMPP CMPP ${ }^{\mathrm{TM}}$; editorial support, including figure preparation, formatting, proofreading, and submission, was provided by Nicola Jenkins, MA (all of Scion, London, UK). This assistance was funded by Merck Sharp \& Dohme Corp., a subsidiary of Merck \& Co., Inc., Kenilworth, NJ, USA. The sponsor was involved in the study design, collection, analysis, and interpretation of data, as well as data checking of information provided in the manuscript. However, the ultimate responsibility for opinions, conclusions, and data interpretation lies with the authors.

Disclosures. George Grunberger: current research contracts from Medtronic, Eli Lilly, Novo Nordisk, AstraZeneca; speakers' bureau: Novo Nordisk, Janssen, Boehringer Ingelheim/ Eli Lilly, Sanofi. Sarah Camp is an employee of
Merck Sharp \& Dohme Corp., a subsidiary of Merck \& Co., Inc., Kenilworth, NJ, USA, and may own stock and/or hold stock options in the company. Jeremy Johnson is an employee of Merck Sharp \& Dohme Corp., a subsidiary of Merck \& Co., Inc., Kenilworth, NJ, USA, and may own stock and/or hold stock options in the company. Susan Huyck is an employee of Merck Sharp \& Dohme Corp., a subsidiary of Merck \& Co., Inc., Kenilworth, NJ, USA, and may own stock and/or hold stock options in the company. Gregory Golm is an employee of Merck Sharp \& Dohme Corp., a subsidiary of Merck \& Co., Inc., Kenilworth, NJ, USA, and may own stock and/or hold stock options in the company. Samuel Engel is an employee of Merck Sharp \& Dohme Corp., a subsidiary of Merck \& Co., Inc., Kenilworth, NJ, USA, and may own stock and/or hold stock options in the company. Brett Lauring is an employee of Merck Sharp \& Dohme Corp., a subsidiary of Merck \& Co., Inc., Kenilworth, NJ, USA, and may own stock and/or hold stock options in the company. Zhi Wei Jiang was an employee of Merck Sharp \& Dohme Corp., a subsidiary of Merck \& Co., Inc., Kenilworth, NJ, USA, at the time the study was conducted. Steven G. Terra is an employee and stock/shareholder in Pfizer Inc. James P. Mancuso is an employee and stock/ shareholder in Pfizer Inc.

Compliance with Ethics Guidelines. All procedures followed were in accordance with the ethical standards of the responsible committee on human experimentation (institutional and national) and with the Helsinki Declaration of 1964, as revised in 2013. Informed consent was obtained from all patients for being included in the study.

Data Availability. Merck \& Co., Inc.'s data sharing policy, including restrictions, is available at http://engagezone.merck.com/ds documentation.php. Requests for access to the study data can be submitted through the EngageZone site or via email to dataaccess@merck.com.

Open Access. This article is distributed under the terms of the Creative Commons 
Attribution-NonCommercial 4.0 International License (http://creativecommons.org/licenses/ by-nc/4.0/), which permits any noncommercial use, distribution, and reproduction in any medium, provided you give appropriate credit to the original author(s) and the source, provide a link to the Creative Commons license, and indicate if changes were made.

Author Contributions Sarah Camp, Jeremy Johnson, Steven G. Terra, James P. Mancuso, Gregory Golm, Sam Engel, and Brett Lauring contributed to the conception, design, or planning of the study. Jeremy Johnson and Brett Lauring contributed to the acquisition of the data. George Grunberger, Jeremy Johnson, Susan Huyck, Zhi Wei Jiang, Gregory Golm, and Brett Lauring contributed to the analysis of the data. George Grunberger, Jeremy Johnson, Susan Huyck, Steven G. Terra, James P. Mancuso, Zhi Wei Jiang, Gregory Golm, Sam Engel, and Brett Lauring contributed to the interpretation of the results. Authors are responsible for the work described in this paper. All authors reviewed the manuscript for important intellectual content and provided final approval of the version to be published. All authors agree to be accountable for all aspects of the work in ensuring that questions related to the accuracy or integrity of any part of the work are appropriately investigated and resolved.

\section{REFERENCES}

1. Vallon V, Thomson SC. Targeting renal glucose reabsorption to treat hyperglycaemia: the pleiotropic effects of SGLT2 inhibition. Diabetologia. 2017;60:215-25.

2. Dandona P, Chaudhuri A. Sodium-glucose co-transporter 2 inhibitors for type 2 diabetes mellitus: an overview for the primary care physician. Int J Clin Pract. 2017;71:e12937.

3. Scheen AJ. Pharmacodynamics, efficacy and safety of sodium-glucose co-transporter type 2 (SGLT2) inhibitors for the treatment of type 2 diabetes mellitus. Drugs. 2015;75:33-59.
4. Fioretto P, Zambon A, Rossato M, Busetto L, Vettor R. SGLT2 inhibitors and the diabetic kidney. Diabetes Care. 2016;39(Suppl 2):S165-71.

5. Kohan DE, Fioretto P, Tang W, List JF. Long-term study of patients with type 2 diabetes and moderate renal impairment shows that dapagliflozin reduces weight and blood pressure but does not improve glycemic control. Kidney Int. 2014;85:962-71.

6. Barnett AH, Mithal A, Manassie J, Jones R, Rattunde $\mathrm{H}$, Woerle HJ, et al. Efficacy and safety of empagliflozin added to existing antidiabetes treatment in patients with type 2 diabetes and chronic kidney disease: a randomised, double-blind, placebo-controlled trial. Lancet Diabetes Endocrinol. 2014;2:369-84.

7. Yale JF, Bakris G, Cariou B, Yue D, David-Neto E, Xi $\mathrm{L}$, et al. Efficacy and safety of canagliflozin in subjects with type 2 diabetes and chronic kidney disease. Diabetes Obes Metab. 2013;15:463-73.

8. Wanner C, Inzucchi SE, Lachin JM, Fitchett D, von Eynatten M, Mattheus M, et al. Empagliflozin and progression of kidney disease in type 2 diabetes. N Engl J Med. 2016;375:323-34.

9. Neal B, Perkovic V, Mahaffey KW, de Zeeuw D, Fulcher G, Erondu N, et al. Canagliflozin and cardiovascular and renal events in type 2 diabetes. N Engl J Med. 2017;377:644-57.

10. Cherney DZ, Perkins BA, Soleymanlou N, Maione M, Lai V, Lee A, et al. Renal hemodynamic effect of sodium-glucose cotransporter 2 inhibition in patients with type 1 diabetes mellitus. Circulation. 2014;129:587-97.

11. Mascitti V, Maurer TS, Robinson RP, Bian J, Boustany-Kari CM, Brandt T, et al. Discovery of a clinical candidate from the structurally unique dioxa-bicyclo[3.2.1] octane class of sodium-dependent glucose cotransporter 2 inhibitors. J Med Chem. 2011;54:2952-60.

12. AstraZeneca Pharmaceuticals. Summary of product characteristics-Forxiga, 2012. http://www.ema. europa.eu/docs/en_GB/document_library/EPAR_Product_Information/human/002322/WC5001360 26.pdf. Accessed July 21, 2017.

13. AstraZeneca Pharmaceuticals. Highlights of prescribing information-FARXIGA ${ }^{\circledR}$ (dapagliflozin) tablets, for oral use 2016. https://www.accessdata. fda.gov/drugsatfda_docs/label/2016/202293s009lbl. pdf. Accessed July 21, 2017.

14. Boehringer Ingelheim Pharmaceuticals I. Highlights of prescribing information-JARDIANCE ${ }^{\circledR}$ (empagliflozin) tablets, for oral use, 2014. https:// 
www.accessdata.fda.gov/drugsatfda_docs/label/2014 /204629s000lbl.pdf. Accessed July 21, 2017.

15. Boehringer Ingelheim Pharmaceuticals I. Summary of product characteristics-Jardiance, 2014. http:// www.ema.europa.eu/docs/en_GB/document_library /EPAR_-_Product_Information/human/002677/WC 500168592.pdf. Accessed July 21, 2017.

16. Janssen Pharmaceuticals I. Highlights of prescribing information-INVOKANA (canagliflozin) tablets, for oral use, 2016. https://www.accessdata.fda.gov/ drugsatfda_docs/label/2016/204042s015s019lbl.pdf. Accessed July 21, 2017.

17. Janssen-Cilag International N. Summary of product characteristics-Invokana 2013. http://www.ema. europa.eu/docs/en_GB/document_library/EPAR_-_ Product_Information/human/002649/WC5001564 56.pdf. Accessed July 21, 2017.

18. American Diabetes Association. Executive summary: standards of medical care in diabetes-2012. Diabetes Care. 2012;35(Suppl 1):S4-10.

19. Liang K-Y, Zeger SL. Longitudinal data analysis of continuous and discrete responses for pre-post designs. Sankhya Ser B. 2000;62:134-48.

20. Miettinen O, Nurminen M. Comparative analysis of two rates. Stat Med. 1985;4:213-26.

21. Gantz I, Sokolova L, Jain L, Iredale C, O’Neill E, Wei $\mathrm{Z}$, et al. Use of prohibited medication, a potentially overlooked confounder in clinical trials: randomized omarigliptin (once-weekly DPP-4 inhibitor) monotherapy trial in 18-45 year-olds. Clin Ther. 2017;9:2024-37.

22. US Food and Drug Administration. FDA Drug Safety Communication: FDA revises warnings regarding use of the diabetes medicine metformin in certain patients with reduced kidney function, 2017. https://www.fda.gov/Drugs/DrugSafety/ucm 493244. htm. Accessed July 21, 2017.

23. Sahasrabudhe V, Terra S, Fountaine R, Hickman A, Saur D, Matschke K, et al. The effect of renal impairment on the pharmacokinetics and pharmacodynamics of ertugliflozin in subjects with type 2 diabetes mellitus. J Clin Pharmacol 2017;57:1432-43.

24. List JF, Woo V, Morales E, Tang W, Fiedorek FT. Sodium-glucose cotransport inhibition with dapagliflozin in type 2 diabetes. Diabetes Care. 2009;32:650-7.

25. Kadokura T, Zhang W, Krauwinkel W, Leeflang S, Keirns J, Taniuchi Y, et al. Clinical pharmacokinetics and pharmacodynamics of the novel SGLT2 inhibitor ipragliflozin. Clin Pharmacokinet. 2014;53:975-88.

26. Macha S, Mattheus M, Halabi A, Pinnetti S, Woerle HJ, Broedl UC. Pharmacokinetics, pharmacodynamics and safety of empagliflozin, a sodium glucose cotransporter 2 (SGLT2) inhibitor, in subjects with renal impairment. Diabetes Obes Metab. 2014;16:215-22.

27. Terra SG, Focht K, Davies M, Frias J, Derosa G, Darekar A, et al. Phase III, efficacy and safety study of ertugliflozin monotherapy in people with type 2 diabetes mellitus inadequately controlled with diet and exercise alone. Diabetes Obes Metab. 2017;19:721-8.

28. Yamout H, Perkovic V, Davies M, Woo V, de Zeeuw D, Mayer C, et al. Efficacy and safety of canagliflozin in patients with type 2 diabetes and stage 3 nephropathy. Am J Nephrol. 2014;40:64-74. 